\title{
Best Linear Unbiased Prediction with Additive Main and Multiplicative Interaction for Stability Analysis of Barley Genotypes Evaluated in Coordinated Program
}

Ajay Verma, R.P.S. Verma, J. Singh, L. Kumar, G.P. Singh

10.18805/IJARe.A-5821

\begin{abstract}
Background: Additive main and multiplicative interaction (AMMI) analysis had been exploited for multi environment trials for most of the crops. Usage of the best linear unbiased prediction (BLUP), along with AMMI tools, of the genotypes would improve the estimation of interaction effects.

Methods: AMMI based measures of adaptability have been enriched with the incorporation of BLUP of genotypes by new Superiority index that allowed variable weights for stability and yield of genotypes.

Result: Stability measure weighted average of absolute scores (WAASB) based on all significant interaction principal components ranked suitability of KB1754, RD3000, NDB1445 genotypes. Superiority index while weighting 0.65 and 0.35 for mean yield and stability arranged DWRB201, NDB1445, RD2552 as of stable high yield performance of barley genotypes. Corrected measure Modified AMMI Stability Value (MASV1) found RD2552, DWRB201, KB1762 and Modified AMMI Stability Value (MASV) ranked DWRB201, RD2552, KB1762. ASTAB measure achieved the desirable lower values for DWRB201 DWRB207, HUB268 genotypes. Biplot graphical analysis based on $60.7 \%$ of variation of the stability measures observed MASV1, ASTAB (AMMI based stability parameter), EV(Averages of the squared eigenvector values), SIPC (Sums of the absolute value of the IPC scores), Za (Absolute value of the relative contribution of IPCs to the interaction), W3, WAASB and MASV had been clubbed together. For the second year lower value of WAASB measure had observed for RD3016, KB1815 HUB273. Ranking of genotypes as per Superiority index found RD3017, RD2907, HUB274 as of stable high yield performance. Genotypes RD3017, RD2907 and NDB1173 pointed out by MASV1 while RD3017, RD2907, NDB1173 identified by MASV as the genotypes of choice. RD3017 NDB1173, RD2907 genotypes were selected as per values of ASTAB measure. Total of $71.8 \%$ of variation of the considered measures in biplot analysis expressed larger cluster comprised of AMMI based measures and a separate cluster of Superiority indexes as per mean, Geometric Adaptability Index (GAI) and HMGV also observed.
\end{abstract}

Key words: AMMI, ASTAB, MASV, SSI, WAASB.

\section{INTRODUCTION}

$\mathrm{G} \times \mathrm{E}$ interaction has been assessed by the differential expression of genotypes over the environments (Ajay et al., 2020). AMMI model explains more information as comprises of additive main effects of genotype and environment and the multiplicative effect of $\mathrm{G} \times \mathrm{E}$ interaction (Gauch, 2013). Research studies observed the better performance of AMMI model than linear regression models and other multivariate procedures (Bocianowski et al., 2019). Several of AMMI based stability measures are available in literature (Zali et al., 2012; Agahi et al., 2020). Researchers have introduced different selection criteria for simultaneous selection of yield and stability (Rao and Prabhakaran 2005; Farshadfar, 2008; Farshadfar et al., 2011). BLUP and $A M M I$, two distinct approaches, utilized to distinguish the pattern from the random error components in $\mathrm{G} \times \mathrm{E}$ interactions (Piepho et al., 2008). The benefits of two important techniques AMMI and BLUP nested into a Superiority Index measure for stability and adaptability of genotypes (Olivoto et al., 2019).

\section{MATERIALS AND METHODS}

Sixteen advanced genotypes at seven locations and eighteen
ICAR-Indian Institute of Wheat and Barley Research, Karnal-132 001, Haryana, India.

Corresponding Author: Ajay Verma, ICAR-Indian Institute of Wheat and Barley Research, Karnal-132 001, Haryana, India. Email: verma.dwr@gmail.com

How to cite this article: Verma, A., Verma, R.P.S., Singh, J., Kumar, L., Singh, G.P. (2021). Best Linear Unbiased Prediction with Additive Main and Multiplicative Interaction for Stability Analysis of Barley Genotypes Evaluated in Coordinated Program. Indian Journal of Agricultural Research. DOI: 10.18805/IJARe.A-5821.

Submitted: 12-05-2021 Accepted: 01-11-2021 Online: 09-12-2021

genotypes at five locations were evaluated under research field trials during 2018-19 and 2019-20 cropping seasons respectively. Field trials were conducted at research centers in randomized complete block designs with four replications. Recommended agronomic practices were followed to harvest good yield. Details of genotype parentage along with environmental conditions were reflected in Table 1 and 2 for ready reference. 
Stability measure weighted average of absolute scores has been calculated as

$$
\text { WAASB }=\sum_{k=1}^{p}\left|I P C A_{i k} \times E P_{k}\right| / \sum_{k=1}^{p} E P_{k}
$$

Where,

WAASB $B_{i}$ was the weighted average of absolute scores of the $i^{\text {th }}$ genotype (or environment); IPCA ik $_{i k}$ was the score of the $i^{\text {th }}$ genotype (or environment) in the $k^{\text {th }} \mathrm{IPCA}$ and $\mathrm{EP}_{k}$ was the amount of the variance explained by the $k^{\text {th }}$ IPCA. Superiority index allowed weighting between yield and stability measures (WAASB) to select genotypes that combined high performance and stability as

Where,

$$
S I=\frac{\left(r G_{i} \times \theta_{Y}\right)+\left(r W_{i} \times \theta_{S}\right)}{\theta_{Y}+\theta_{S}}
$$

$r G_{i}$ and $r W_{i}$ were the rescaled values for yield and WAASB, respectively, for the $i^{\text {th }}$ genotype; $G_{i}$ and $W_{i}$ were the yield and the WAASB values for $i^{\text {th }}$ genotype. SI superiority index for the $i^{\text {th }}$ genotype that weights between yield and stability and $\theta_{Y}$ and $\theta_{S}$ were the weights for yield and stability assumed to be of order 65 and 35 respectively in this study,

Zobel 1994

Averages of the squared eigenvector values

$E V=\sum_{n=1}^{n} \lambda_{\text {in }}^{2} / n$

Sneller

et al., 1997

Sums of the absolute

value of the IPC scores

$\mathrm{SIPC}=\sum_{\mathrm{n}=1}^{\mathrm{n}} \lambda_{n}^{0.5} \gamma_{\mathrm{in}}$

Rao and AMMI based stability

Prabhakaran, parameter

2005

$\mathrm{ASTAB}=\sum_{n=1}^{n} \lambda_{n} \gamma_{\mathrm{ni}}^{2}$

Zali et al., Modified AMMI stability value

2012

MASV $=\sqrt{\sum_{n=1}^{n-1} \frac{\text { SSIPC }_{n}}{\operatorname{SSIPC}_{n+1}}\left(P C_{n}\right)^{2}+\left(P C_{n+1}\right)^{2}}$

Zali et al., 2012

Absolute value of the relative contribution of $\quad Z_{a}=\sum_{n=1}^{N}\left|\lambda_{n} \gamma_{\text {in }}\right|$ IPCs to the interaction

Ajay et al., 2020

MASV $1=\sqrt{\sum_{n=1}^{n-1} \frac{\text { SSIPC }_{n}}{\operatorname{SSIPC}_{n+1}}\left(P C_{n}\right)^{2}+\left(P C_{n+1}\right)^{2}}$

Resende and Relative performance of

Durate, 2007 genotypic values across $P R V G_{i j}=V G_{i j} / V G_{i}$ environments

Resende and Harmonic mean of Relative performance of Durate, 2007 genotypic values

$\mathrm{MHPRVG}_{\mathrm{i}}=$ Number of environments /

$$
\sum_{j=1}^{k} \frac{1}{\text { PRVGij }}
$$

$\begin{array}{ll}\text { Olivato et al., } & \begin{array}{l}\text { Superiority } \\ \text { Index }\end{array} 019\end{array} \quad \mathrm{SI}=\frac{\left(r G_{i} \times \theta_{\mathrm{Y}}\right)+\left(r \mathrm{rW}_{\mathrm{i}} \times \theta_{\mathrm{S}}\right)}{\theta_{\mathrm{Y}}+\theta_{\mathrm{S}}}$

AMMI analysis was performed using AMMISOFT version 1.0, available at https://scs.cals.cornell.edu/people/ hugh-gauch/ and SAS software version 9.3.

\section{RESULTS AND DISCUSSION AMMI analysis of barley genotypes}

\section{First year of study 2018-19}

Highly significant effects of environment $(E), G \times E$ interaction and genotypes (G) had been observed by AMMI analysis. Environment explained about $31.8 \%$ of the total sum of squares due to treatments significantly indicated diverse environments caused most of the variations in genotypes yield (Table 3 ). Significant proportion of $\mathrm{G} \times \mathrm{E}$ interaction deserved the stability estimation of genotypes over environments (Ajay et al., 2020). Genotypes explained only $9.3 \%$ of total sum of squares, whereas $\mathrm{G} \times \mathrm{E}$ interaction accounted for $38.4 \%$ of treatment variations in yield. More of $\mathrm{G} \times \mathrm{E}$ interaction sum of squares as compared to genotypes indicated the presence of genotypic differences across environments and complex $\mathrm{G} \times \mathrm{E}$ interaction for wheat yield. Partitioning of $G \times E$ interaction revealed that the first five multiplicative terms (IPCA1, IPCA2, IPCA3, IPCA4 and IPCA5) of AMMI were significant and explained $35.4 \%$, $29.6 \%, 25.7 \%, 5.7 \%$ and $2.8 \%$ of interaction sum of squares, respectively. Total of significant components were $99.2 \%$ and remaining $0.8 \%$ was the residual or noise that discarded (Oyekunle et al., 2017).

\section{Second year of study $\mathbf{2 0 1 9 - 2 0}$}

Highly significant effects of environment $(E), G \times E$ interaction and genotypes (G) had been observed by AMMI analysis. Environment explained about significantly $42.5 \%$ of the total sum of squares due to treatments indicating that diverse environments caused most of the variations in genotypes yield (Table 7). G×E interaction accounted for $30.3 \%$ whereas Genotypes contributed only $8.4 \%$ of total treatment variations in yield. Further analysis of $\mathrm{G} \times \mathrm{E}$ interaction observed three multiplicative terms (IPCA1, IPCA2 and IPCA3) explained $49.2 \%, 24.3 \%$ and $19.7 \%$ of interaction sum of squares, respectively. Total of these components were to the tune of $93.2 \%$ and remaining was noise that was discarded.

\section{Ranking of barley genotypes as per AMMI based stability measures}

\section{First year of study 2018-19}

Least value of absolute IPCA1 expressed by HUB268, DWRB201, RD2999 and higher value achieved by RD3002 (Table 4). Low values of (EV) associated with stable behaviour, the genotypes HUB267 followed by RD2999, NDB1173 expressed lower values and maximum value by KB1754 genotype. Measure SIPC identified HUB267 followed by RD2999, DWRB201 as of stable nature, whereas KB1754 would be of least stable type. Za measure considered absolute value of the relative contribution of IPCs to the interaction revealed DWRB201, HUB267 and RD2999 as genotypes with descending order of stability, whereas KB1754 genotype with the least stability. ASTAB measure observed genotypes HUB267 and DWRB201 as stable and 
KB1754 was least stable in this study (Rao and Prabhakaran 2005). All significant IPCAs had been considered by MASV and MASV1 measures. Values of MASV1 showed that the genotypes, RD2552, DWRB201 and RD2999 were most stable and DWRB201, HUB267 and RD2999 would be stable by MASV measure respectively (Ajay et al., 2020). Measure W1 favoured RD3002 RD2552, RD3002 while as per W2, genotypes identified were RD3000, RD2552, RD3002 while W3 favoured RD3000, KB1754, RD3002 whereas W4 settled for KB1754, RD3000, NDB1445. Finally lower values of WAASB associated with stable nature of
KB1754, RD3000, NDB1445 genotypes as for considered locations of the zone at the same time maximum deviation from the average performance across environments obtained by DWRB201.

\section{Second year of study 2019-20}

Least absolute values of IPCA1 expressed by RD2907, HUB274, KB1845 and higher value achieved by KB1815 (Table 8). Minimum values of EV associated with stable behaviour of RD3015, NDB1173, RD2907 genotypes and maximum value had by RD3016 genotype. SIPC measure

Table 1: Parentage details of barley genotypes and environmental conditions (2018-19).

\begin{tabular}{|c|c|c|c|c|c|c|}
\hline Code & Genotype & Parentage & Code & Location & Latitude & Longitude \\
\hline G1 & RD2794 & RD2035/RD2683 & $\mathrm{E} 1$ & IIWBR Hisar & $29^{\circ} 14^{\prime} \mathrm{N}$ & $75^{\circ} 73^{\prime} \mathrm{E}$ \\
\hline G2 & HUB267 & BH 550/RD 2624 & E2 & Faizabad & $26^{\circ} 46^{\prime} \mathrm{N}$ & $82^{\circ} 9^{\prime} \mathrm{E}$ \\
\hline G3 & RD2999 & RD2592/RD2830 & E3 & Khumer & $25^{\circ} 42^{\prime} \mathrm{N}$ & $93^{\circ} 96^{\prime} \mathrm{E}$ \\
\hline G4 & NDB1708 & 3rd GSBYT-18 (2016) & E4 & Dalipnagar & $28^{\circ} 63^{\prime} \mathrm{N}$ & $77^{\circ} 21^{\prime} \mathrm{E}$ \\
\hline G5 & DWRB207 & CDC MANLEY/BCU2881 & E5 & Banasthali & $26^{\circ} 40^{\prime} \mathrm{N}$ & $75^{\circ} 85^{\prime} \mathrm{E}$ \\
\hline G6 & KB1762 & PENCO/CHEVRON-BAR//KASOTA & E6 & HAU Hisar & $29^{\circ} 10^{\prime} \mathrm{N}$ & $75^{\circ} 46^{\prime} \mathrm{E}$ \\
\hline G7 & DWRB201 & DWRUB52/BONMRA-73//Prestige/PL426 & E7 & Vallabhnagar & $24^{\circ} 67^{\prime} \mathrm{N}$ & $74^{\prime} \mathrm{E}$ \\
\hline \multirow[t]{2}{*}{ G8 } & KB1754 & LIGNEE527/GERBEL/3/BOY-B²/SURB// & & & & \\
\hline & & Cl12225.2D/4/BBSC/CONG0NA & & & & \\
\hline G9 & HUB268 & YARADU/22NDIBYT-01-2-2-4-2 & & & & \\
\hline G10 & KB1706 & Jagriti/RD2785 & & & & \\
\hline G11 & NDB1173 & BYTLRA 3-(1994-95)/NDB217 & & & & \\
\hline G12 & RD3000 & DWRUB64/RD2503 & & & & \\
\hline G13 & RD2552 & RD2035/DL472 & & & & \\
\hline G14 & RD3002 & RD2715/RD2552 & & & & \\
\hline G15 & NDB1445 & NDB940/Ratna & & & & \\
\hline G16 & RD2907 & RD103/RD2518//RD2592 & & & & \\
\hline
\end{tabular}

Table 2: Parentage details of barley genotypes and environmental conditions (2019-20).

\begin{tabular}{|c|c|c|c|c|c|c|}
\hline Code & Genotype & Parentage & Code & Location & Latitude & Longitude \\
\hline$\overline{\mathrm{G} 1}$ & KB1845 & $\begin{array}{l}\text { PETUNIA1/5/POST/COPAL//GLORIA-BAR/COME/3/SIND89A- } \\
\text { 148/4/CARD/6/GLORIA-BAR/COPAL//BLLU/3/PETUNIA 1/7/PINON }\end{array}$ & $\mathrm{E} 1$ & IIWBR, Hisar & $29^{\circ} 14^{\prime} \mathrm{N}$ & $75^{\circ} 73^{\prime} \mathrm{E}$ \\
\hline G2 & DWRB214 & EC361898 & E2 & HAU, Hisar & $29^{\circ} 10^{\prime} \mathrm{N}$ & $75^{\circ} 46^{\prime} \mathrm{E}$ \\
\hline G3 & RD3017 & RD 2552/PL 419//RD 2508 & E3 & Dalipnagar & $28^{\circ} 63^{\prime} \mathrm{N}$ & $77^{\circ} 21^{\prime} \mathrm{E}$ \\
\hline G4 & HUB274 & JB $18 / 31^{\text {st }}$ IBON-4-02 & $\mathrm{E} 4$ & Faizabad & $26^{\circ} 46^{\prime} \mathrm{N}$ & $82^{\circ} 9^{\prime} \mathrm{E}$ \\
\hline G5 & $\mathrm{BH} 1033$ & BH 942/BH 393 & E5 & Fatehpur & $25^{\circ} 93^{\prime} \mathrm{N}$ & $80^{\circ} 81^{\prime} \mathrm{E}$ \\
\hline G6 & RD2794 & RD2035/RD2683 & & & & \\
\hline G7 & RD3018 & RD 2592/RD 2607 & & & & \\
\hline G8 & RD2907 & RD103/RD2518//RD2592 & & & & \\
\hline G9 & NDB1730 & Avt/Attiki//M-AH73-337-1/3/Aths/Lignee686/4/HB42 & & & & \\
\hline G10 & KB1822 & K 996/K 508 & & & & \\
\hline G11 & NDB1742 & Avt/Attiki//M-AH73-337-1/3/Aths/Lignee686/4/HB42 & & & & \\
\hline G12 & $\mathrm{BH} 1032$ & BH 965/BH 885 & & & & \\
\hline G13 & RD3016 & RD 2715/RD 2552 & & & & \\
\hline G14 & NDB1173 & BYTLRA 3-(1994-95)/NDB217 & & & & \\
\hline G15 & HUB273 & 31st INBON-18/RD 2508 & & & & \\
\hline G16 & KB1815 & $\begin{array}{l}\text { Ghinneri(smooth_awns)/6/JLB70-01/5/DeirAlla106//DL70/Pyo/3/RM } \\
\text { 1508/4/Arizona5908/Aths//Avt/Attiki/3/Ager }\left(4^{\text {th }} \text { GSBSN2016-17-52) }\right.\end{array}$ & & & & \\
\hline G17 & RD3015 & RD 2715/RD 2552 & & & & \\
\hline G18 & RD2552 & RD2035/DL472 & & & & \\
\hline
\end{tabular}


Best Linear Unbiased Prediction with Additive Main and Multiplicative Interaction for Stability Analysis of Barley Genotypes...

Table 3: AMMI analysis and percentage contribution of significant interaction principal components (2018-19).

\begin{tabular}{|c|c|c|c|c|c|c|}
\hline Source & $\begin{array}{l}\text { Degree of } \\
\text { freedom }\end{array}$ & $\begin{array}{c}\text { Mean sum of } \\
\text { squares }\end{array}$ & $\begin{array}{c}\text { Level of } \\
\text { significance }\end{array}$ & $\begin{array}{c}\text { Proportional } \\
\text { contributionof factors }\end{array}$ & $\begin{array}{c}\mathrm{G} \times \mathrm{E} \text { interaction sum } \\
\text { of squares (\%) }\end{array}$ & $\begin{array}{l}\text { Cumulative sum of squares } \\
\text { (\%) by IPCA's }\end{array}$ \\
\hline Treatments & 111 & 237.21 & $.0000000^{* * *}$ & 79.49 & & \\
\hline Genotype (G) & 15 & 205.41 & $.0000000^{* * *}$ & 9.30 & & \\
\hline Environment (E) & 6 & 1753.76 & $.0000000^{* * *}$ & 31.77 & & \\
\hline $\mathrm{G} \times \mathrm{E}$ interactions & 90 & 141.40 & $.0000000^{* * *}$ & 38.42 & & \\
\hline IPC1 & 20 & 225.05 & $.0000000^{* * *}$ & & 35.37 & 35.37 \\
\hline IPC2 & 18 & 209.27 & $.0000000^{* * *}$ & & 29.60 & 64.97 \\
\hline IPC3 & 16 & 204.18 & $.0000000^{* * *}$ & & 25.67 & 90.64 \\
\hline IPC4 & 14 & 51.54 & $.0038716^{* *}$ & & 5.67 & 96.31 \\
\hline IPC5 & 12 & 30.26 & 0.2688757 & & 2.85 & 99.16 \\
\hline Residual & 10 & 10.69 & 0.8241293 & & & \\
\hline Error & 336 & 20.22 & & & & \\
\hline Total & 447 & 74.10 & & & & \\
\hline
\end{tabular}

Table 4: AMMI based measures and Weighted average of absolute scores for barley genotypes 2018-19.

\begin{tabular}{lcccccccccccc}
\hline Genotype & IPCA1 & EV & SIPC & Za & ASTAB & MASV1 & MASV & W1 & W2 & W3 & W4 & WAASB \\
\hline RD2794 & 0.9777 & 0.0353 & 5.7968 & 16.88 & 51.42 & 7.94 & 4.89 & 0.9777 & 1.1743 & 1.2685 & 1.2833 & 1.2503 \\
HUB267 & 0.2403 & 0.0115 & 2.8172 & 7.55 & 13.64 & 5.26 & 2.83 & 0.2403 & 0.2850 & 0.5448 & 0.5365 & 0.5439 \\
RD2999 & 0.2141 & 0.0183 & 3.3412 & 10.59 & 36.33 & 3.67 & 3.22 & 0.2141 & 1.1035 & 0.8731 & 0.8142 & 0.8052 \\
NDB1708 & 1.0327 & 0.0513 & 6.0237 & 15.71 & 57.97 & 6.06 & 4.76 & 1.0327 & 1.3415 & 1.0835 & 1.1605 & 1.1398 \\
DWRB207 & 0.4052 & 0.0339 & 4.7014 & 11.57 & 34.39 & 7.72 & 4.49 & 0.4052 & 0.4110 & 0.7527 & 0.8079 & 0.8121 \\
KB1762 & 1.3091 & 0.0311 & 5.4706 & 15.47 & 42.41 & 5.46 & 3.73 & 1.3091 & 1.2953 & 1.1760 & 1.1567 & 1.1519 \\
DWRB201 & 0.1872 & 0.0461 & 3.6856 & 6.87 & 22.99 & 3.72 & 2.70 & 0.1872 & 0.2484 & 0.3624 & 0.3788 & 0.4468 \\
KB1754 & 0.8507 & 0.0516 & 6.5966 & 22.71 & 110.33 & 15.30 & 8.11 & 0.8507 & 1.2800 & 1.9059 & 1.7874 & 1.7311 \\
HUB268 & 0.1596 & 0.0227 & 3.7002 & 10.65 & 32.07 & 8.37 & 4.60 & 0.1596 & 0.3261 & 0.7637 & 0.8002 & 0.7703 \\
KB1706 & 0.9500 & 0.0213 & 4.5730 & 13.71 & 35.44 & 4.48 & 3.29 & 0.9500 & 1.2648 & 1.0868 & 1.0457 & 1.0343 \\
NDB1173 & 1.9334 & 0.0206 & 4.4705 & 15.88 & 50.38 & 7.07 & 4.11 & 1.9334 & 1.3540 & 1.3733 & 1.2914 & 1.2420 \\
RD3000 & 2.3994 & 0.0336 & 5.6970 & 20.38 & 83.85 & 6.15 & 4.51 & 2.3994 & 2.1688 & 1.7903 & 1.6629 & 1.6059 \\
RD2552 & 2.5236 & 0.0276 & 4.5816 & 15.23 & 64.40 & 3.62 & 3.32 & 2.5236 & 1.8587 & 1.3130 & 1.2250 & 1.1999 \\
RD3002 & 2.5512 & 0.0278 & 5.0773 & 16.85 & 65.53 & 5.24 & 3.72 & 2.5512 & 1.6984 & 1.4293 & 1.3496 & 1.3150 \\
NDB1445 & 0.3770 & 0.0371 & 5.3830 & 17.45 & 75.97 & 7.13 & 5.17 & 0.3770 & 1.5283 & 1.4060 & 1.3732 & 1.3209 \\
RD2907 & 2.3109 & 0.0303 & 5.0677 & 16.56 & 65.33 & 7.87 & 4.57 & 2.3109 & 1.2986 & 1.3873 & 1.3052 & 1.2791 \\
\hline
\end{tabular}

Table 5: Superiority index measures and corresponding ranking of genotypes 2018-19.

\begin{tabular}{lcccccccccccccccccccc}
\hline Genotype & IPCA1 & EV & SIPC & Za & ASTAB & MASV1 & MASV & Mean & $R_{k}$ & Slam & $R_{k}$ & $G_{M}$ & $R_{k}$ & SIgm & $R_{k}$ & $H M$ & $R_{k}$ & SIhm & $R_{k}$ \\
\hline RD2794 & 24 & 27 & 29 & 28 & 24 & 29 & 29 & 31.60 & 15 & 29.57 & 14 & 31.21 & 15 & 30.51 & 14 & 30.81 & 14 & 31.78 & 13 \\
HUB267 & 13 & 10 & 10 & 11 & 10 & 15 & 11 & 33.83 & 9 & 65.05 & 6 & 33.40 & 8 & 66.89 & 6 & 32.96 & 7 & 69.31 & 5 \\
RD2999 & 14 & 13 & 13 & 14 & 17 & 13 & 14 & 32.68 & 11 & 49.56 & 8 & 32.11 & 12 & 49.66 & 8 & 31.50 & 11 & 49.82 & 9 \\
NDB1708 & 20 & 25 & 25 & 19 & 20 & 18 & 23 & 33.31 & 10 & 45.03 & 10 & 32.60 & 9 & 44.34 & 10 & 31.89 & 10 & 44.03 & 10 \\
DWRB207 & 11 & 16 & 13 & 10 & 9 & 17 & 14 & 35.70 & 5 & 71.35 & 4 & 35.23 & 5 & 73.82 & 3 & 34.73 & 4 & 77.07 & 2 \\
KB1762 & 15 & 13 & 16 & 12 & 11 & 11 & 11 & 36.51 & 4 & 68.01 & 5 & 35.53 & 4 & 66.94 & 5 & 34.46 & 5 & 65.51 & 6 \\
DWRB201 & 5 & 17 & 6 & 4 & 5 & 6 & 4 & 36.80 & 3 & 89.32 & 1 & 36.22 & 3 & 91.48 & 1 & 35.54 & 2 & 93.92 & 1 \\
KB1754 & 21 & 30 & 30 & 30 & 30 & 30 & 30 & 32.55 & 14 & 23.37 & 15 & 31.36 & 14 & 18.55 & 15 & 30.17 & 15 & 13.29 & 15 \\
HUB268 & 8 & 12 & 11 & 11 & 10 & 22 & 19 & 34.04 & 7 & 60.38 & 7 & 33.60 & 6 & 62.27 & 7 & 33.12 & 6 & 64.52 & 7 \\
KB1706 & 20 & 16 & 18 & 18 & 17 & 16 & 16 & 32.65 & 12 & 43.11 & 11 & 31.81 & 13 & 41.11 & 11 & 30.96 & 12 & 38.95 & 12 \\
NDB1173 & 18 & 9 & 11 & 16 & 14 & 16 & 14 & 34.16 & 6 & 48.46 & 9 & 33.56 & 7 & 49.12 & 9 & 32.92 & 8 & 49.98 & 8 \\
RD3000 & 22 & 18 & 21 & 23 & 23 & 17 & 18 & 33.88 & 8 & 36.47 & 12 & 32.46 & 10 & 30.55 & 13 & 30.87 & 13 & 22.65 & 14 \\
RD2552 & 17 & 8 & 9 & 9 & 13 & 3 & 7 & 37.59 & 2 & 74.58 & 3 & 36.40 & 2 & 72.40 & 4 & 35.18 & 3 & 70.28 & 4 \\
RD3002 & 32 & 23 & 26 & 28 & 29 & 21 & 22 & 29.35 & 16 & 11.34 & 16 & 28.98 & 16 & 11.34 & 16 & 28.61 & 16 & 11.34 & 16 \\
NDB1445 & 6 & 14 & 12 & 15 & 15 & 12 & 16 & 38.26 & 1 & 76.18 & 2 & 37.31 & 1 & 76.18 & 2 & 36.26 & 1 & 76.18 & 3 \\
RD2907 & 26 & 21 & 22 & 24 & 25 & 26 & 24 & 32.56 & 13 & 35.77 & 13 & 32.29 & 11 & 38.13 & 12 & 32.00 & 9 & 41.16 & 11 \\
\hline
\end{tabular}

$\mathrm{AMu}, \mathrm{GMu}, \mathrm{HMu}=$ Arithmetic, Geometric, Harmonic Mean for BLUP values; SI au, SI gu, SI hu= Superiority index as per Arithmetic, Geometric, Harmonic Mean; RPGVu, MHRPGVu= Relative performance and Harmonic mean of Relative Performance as per BLUP of genotypes; Rk= Rank of genotypes. 
identified RD2907, NDB1173 followed by RD3015 for the lower value, whereas RD3016 would be of least stable behaviour. Za measure revealed RD2907, NDB1173 and RD3015 genotypes in descending order of stability, whereas RD3016 genotype with the least stability. ASTAB measure observed genotypes NDB1173, RD2907 and RD3015 as most stable and genotype RD3016 was least stable in this study (Rao and Prabhakaran 2005). RD2907, NDB1173, RD3015 genotypes were of choice by of MASV1 and MASV measure observed RD2907, NDB1173, RD3015 as the

Table 6: Loadings of measures as per two principal components 2018-19.

\begin{tabular}{lcc}
\hline Measure & PC1 & PC2 \\
\hline IPCA1 & 0.1984 & -0.3679 \\
MASV1 & 0.1784 & 0.3560 \\
MASV & 0.2193 & 0.3480 \\
Za & 0.3050 & 0.0561 \\
EV & 0.1151 & 0.2694 \\
SIPC & 0.2623 & 0.1239 \\
ASTAB & 0.2855 & 0.1199 \\
W1 & 0.1984 & -0.3679 \\
W2 & 0.2424 & -0.1927 \\
W3 & 0.3035 & 0.0063 \\
W4 & 0.3051 & 0.0231 \\
WAASB & 0.3059 & 0.0244 \\
Hisar & -0.1241 & -0.2077 \\
Faizabad & -0.0608 & 0.2917 \\
Khumer & -0.1450 & 0.0957 \\
Dalipnagar & -0.0292 & -0.0485 \\
Banasthali & 0.0693 & -0.0162 \\
Hisar & -0.0385 & -0.3127 \\
Vallabhnagar & -0.0047 & 0.2537 \\
Siam & -0.2459 & 0.1196 \\
Sigm & -0.2587 & 0.1085 \\
Sihm & -0.2694 & 0.0974 \\
60.72 & 45.72 & 14.50 \\
\hline
\end{tabular}

stable genotypes while KB1815 would be unstable (Ajay et al., 2019). W1 measure selected KB1815, BH1032, RD2794 while measure W2 favoured KB1815, RD3016, HUB273 barley genotypes. Lower value of WAASB measure had observed for RD3016, KB1815, HUB273 and large value by RD2907.

\section{Superiority indexes as per AMMI and BLUP: Barley} genotypes

\section{First year of study 2018-19}

Stability alone is not a desirable selection criterion as stable genotypes may not be a high yielder, simultaneous use of yield and stability in a single measure is essential (Kang 1993; Farshadar et al., 2008). Simultaneous Selection Index also referred to as genotype stability index (GSI) or yield stability index (YSI) (Farshadar et al., 2011) was computed by adding the ranks of stability measure and mean yield of genotypes. Least ranks for IPCA1 measure exhibited by DWRB201, NDB1445 and HUB268 were considered as stable with high yield, whereas high values suggested as least stable yield for RD3002 genotype (Table 5). EV measure identified RD2552, NDB1173 andHUB267 whereas SPIC favoured DWRB201, RD2552 and HUB267genotypes. Genotypes DWRB201, RD2552 and DWRB207 possessed lower value of $Z$ a measure. ASTAB measure achieved the desirable lower values for DWRB201, DWRB207, HUB268. Composite measure MASV1 found RD2552, DWRB201, KB1762 and as per MASV ranks DWRB201, RD2552, KB1762 genotypes would be of choice for these locations of the zone.

Average yield of genotypes favoured NDB1445, RD2552, DWRB201 where Geometric adaptability index selected NDB1445, RD2552, DWRB201 while Harmonic mean of yield values pointed for NDB1445, DWRB201, RD2552 as suitable genotypes as far as considered locations are concerned. Superiority index while weighting 0.65 and 0.35 for average yield and stability found DWRB201, NDB1445 and RD2552 as of stable performance with high yield. Least magnitude of Slgm ranked DWRB201, NDB1445, DWRB207 as desirable

Table 7: AMMI analysis and percentage contribution of significant interaction principal components (2019-20).

\begin{tabular}{|c|c|c|c|c|c|c|}
\hline Source & $\begin{array}{l}\text { Degree } \\
\text { of } \\
\text { freedom }\end{array}$ & $\begin{array}{c}\text { Mean } \\
\text { sum of } \\
\text { squares }\end{array}$ & $\begin{array}{c}\text { Level } \\
\text { of } \\
\text { significance }\end{array}$ & $\begin{array}{l}\text { Proportional } \\
\text { contribution } \\
\text { of factors }\end{array}$ & $\begin{array}{c}\mathrm{G} \times \mathrm{E} \text { interaction } \\
\text { sum of } \\
\text { squares (\%) }\end{array}$ & $\begin{array}{c}\text { Cumulative sum } \\
\text { of squares(\%) } \\
\text { by IPCA's }\end{array}$ \\
\hline Treatments & 89 & 160.59 & $.0000000^{* * *}$ & 81.17 & & \\
\hline Genotype (G) & 17 & 87.44 & $.0000000^{* * *}$ & 8.44 & & \\
\hline Environment (E) & 4 & 1868.68 & $.0000000^{* * *}$ & 42.45 & & \\
\hline $\mathrm{G} \times \mathrm{E}$ interactions & 68 & 78.40 & $.0000000 * * *$ & 30.28 & & \\
\hline IPC1 & 20 & 131.18 & $.0000000^{* * *}$ & & 49.21 & 49.21 \\
\hline IPC2 & 18 & 71.82 & $.0000000 * * *$ & & 24.25 & 73.46 \\
\hline IPC3 & 16 & 65.62 & $.0000721^{* * *}$ & & 19.69 & 93.16 \\
\hline Residual & 14 & 26.06 & 0.150117 & & & \\
\hline Error & 180 & 18.42 & & & & \\
\hline Total & 269 & 65.46 & & & & \\
\hline
\end{tabular}


genotypes while SIhm measure favoured DWRB201, DWRB207, NDB1445 barley genotypes.

\section{Second year of study 2019-20}

Ranks for IPCA1 measure favoured HUB274, RD2907, RD3017 as per the least values, whereas large values of KB1815 suggested unstable high yield (Table 9). EV measure settled for RD3017, NDB1173 and HUB274 genotypes. Minimum ranks of SPIC favoured RD3017, RD2907 andHUB274 genotypes. Lower value of Za measure possessed by RD3017, HUB274 andRD2907 genotypes for stable higher yield as compared to others genotypes. Barley genotypes RD3017, NDB1173, RD2907 were selected as per values of ASTAB measure accounted AMMI analysis with BLUP of genotypes yield values. Composite measure MASV1 selected RD3017, RD2907, NDB1173 while RD3017, RD2907, NDB1173 identified by MASV as genotypes of choice for these locations of the zone. Superiority index while weighting 0.65 and 0.35 for GAI and stability found RD3017, RD2907 and HUB274 as of stable performance with high yield. While considering Harmonic mean and stability identified RD2907, RD3017, NDB1173 barley genotypes.

Table 8: AMMI based measures and Weighted average of absolute scores of barley genotypes 2019-20.

\begin{tabular}{|c|c|c|c|c|c|c|c|c|c|c|}
\hline Genotype & IPCA1 & EV & SIPC & $\mathrm{Za}$ & ASTAB & MASV1 & MASV & W1 & W2 & WAASB \\
\hline KB1845 & 0.2330 & 0.061 & 3.648 & 16.48 & 35.36 & 3.03 & 2.92 & 0.2330 & 0.6405 & 0.9801 \\
\hline DWRB214 & 0.5716 & 0.021 & 2.128 & 10.81 & 14.45 & 2.53 & 2.27 & 0.5716 & 0.8698 & 0.6919 \\
\hline RD3017 & 0.5655 & 0.008 & 1.368 & 7.43 & 5.92 & 1.68 & 1.41 & 0.5655 & 0.6405 & 0.4902 \\
\hline HUB274 & 0.1808 & 0.017 & 1.986 & 9.18 & 10.81 & 2.01 & 1.89 & 0.1808 & 0.5346 & 0.5565 \\
\hline $\mathrm{BH} 1033$ & 0.4907 & 0.012 & 1.941 & 9.73 & 8.53 & 1.87 & 1.65 & 0.4907 & 0.6512 & 0.6157 \\
\hline RD2794 & 1.9216 & 0.034 & 3.300 & 19.13 & 32.01 & 4.09 & 2.99 & 1.9216 & 1.4597 & 1.2894 \\
\hline RD3018 & 1.4636 & 0.044 & 3.498 & 18.78 & 32.04 & 3.46 & 2.73 & 1.4636 & 1.0737 & 1.2201 \\
\hline RD2907 & 0.0184 & 0.006 & 0.850 & 3.71 & 3.42 & 0.78 & 0.78 & 0.0184 & 0.0331 & 0.2136 \\
\hline NDB1730 & 0.6049 & 0.026 & 2.791 & 13.68 & 16.62 & 2.33 & 2.10 & 0.6049 & 0.7463 & 0.8526 \\
\hline KB1822 & 1.4944 & 0.031 & 2.854 & 16.25 & 26.66 & 3.70 & 2.92 & 1.4944 & 1.4378 & 1.0926 \\
\hline NDB1742 & 0.3350 & 0.038 & 2.806 & 13.01 & 21.70 & 2.20 & 2.11 & 0.3350 & 0.4612 & 0.7832 \\
\hline $\mathrm{BH} 1032$ & 2.1386 & 0.043 & 3.428 & 20.33 & 41.47 & 4.74 & 3.54 & 2.1386 & 1.8079 & 1.3872 \\
\hline RD3016 & 1.6569 & 0.092 & 5.518 & 28.25 & 64.81 & 5.16 & 4.41 & 1.6569 & 1.8678 & 1.8045 \\
\hline NDB1173 & 0.3316 & 0.004 & 0.905 & 4.81 & 2.59 & 1.10 & 0.95 & 0.3316 & 0.4082 & 0.3146 \\
\hline HUB273 & 1.8888 & 0.061 & 4.623 & 24.91 & 49.09 & 4.81 & 3.85 & 1.8888 & 1.8272 & 1.6300 \\
\hline KB1815 & 2.8812 & 0.059 & 3.842 & 23.83 & 62.47 & 5.90 & 4.18 & 2.8812 & 1.9649 & 1.6484 \\
\hline RD3015 & 0.7494 & 0.004 & 0.952 & 6.01 & 4.16 & 1.54 & 1.09 & 0.7494 & 0.5327 & 0.4187 \\
\hline RD2552 & 0.4384 & 0.017 & 2.120 & 10.30 & 10.51 & 1.68 & 1.53 & 0.4384 & 0.4761 & 0.6375 \\
\hline
\end{tabular}

Table 9: Superiority index measures and corresponding ranking of genotypes 2019-20.

\begin{tabular}{lccccccccccccccccccc}
\hline Genotype & IPCA1 & EV & SIPC & Za & ASTAB & MASV1 & MASV & Mean & $R_{k}$ & Slam & $R_{k}$ & $G M$ & $R_{k}$ & SIgm & $R_{k}$ & $H_{M}$ & $R_{k}$ & SIhm & $R_{k}$ \\
\hline KB1845 & 21 & 34 & 33 & 30 & 32 & 29 & 31 & 22.22 & 18 & 18.14 & 18 & 21.97 & 18 & 18.14 & 17 & 21.73 & 15 & 20.60 & 16 \\
DWRB214 & 22 & 21 & 21 & 21 & 21 & 23 & 23 & 26.20 & 13 & 49.88 & 13 & 25.01 & 15 & 43.90 & 13 & 23.79 & 14 & 39.63 & 12 \\
RD3017 & 11 & 7 & 7 & 7 & 7 & 8 & 7 & 29.31 & 3 & 74.15 & 1 & 28.58 & 3 & 71.13 & 1 & 27.72 & 3 & 68.21 & 2 \\
HUB274 & 7 & 11 & 11 & 10 & 12 & 12 & 12 & 28.62 & 5 & 68.33 & 3 & 28.02 & 4 & 66.06 & 3 & 27.29 & 5 & 64.10 & 6 \\
BH1033 & 21 & 19 & 19 & 20 & 19 & 20 & 20 & 26.13 & 14 & 51.15 & 11 & 25.49 & 12 & 48.65 & 11 & 24.93 & 12 & 48.32 & 11 \\
RD2794 & 22 & 17 & 18 & 20 & 18 & 20 & 20 & 28.46 & 6 & 51.18 & 10 & 28.01 & 5 & 49.87 & 10 & 27.52 & 4 & 49.40 & 10 \\
RD3018 & 20 & 22 & 22 & 21 & 21 & 20 & 19 & 28.00 & 8 & 49.76 & 14 & 26.66 & 9 & 42.82 & 14 & 25.03 & 11 & 35.60 & 13 \\
RD2907 & 10 & 12 & 10 & 10 & 11 & 10 & 10 & 27.90 & 9 & 71.29 & 2 & 27.42 & 6 & 69.77 & 2 & 26.92 & 6 & 69.40 & 1 \\
NDB1730 & 14 & 13 & 13 & 14 & 13 & 13 & 12 & 28.67 & 4 & 62.09 & 7 & 27.26 & 7 & 54.69 & 7 & 26.03 & 8 & 49.84 & 9 \\
KB1822 & 15 & 12 & 13 & 13 & 13 & 15 & 14 & 30.08 & 2 & 65.82 & 4 & 29.68 & 2 & 64.88 & 6 & 29.30 & 2 & 64.67 & 5 \\
NDB1742 & 12 & 19 & 17 & 16 & 17 & 15 & 16 & 28.40 & 7 & 61.91 & 8 & 25.46 & 13 & 44.76 & 12 & 21.48 & 16 & 23.44 & 15 \\
BH1032 & 28 & 24 & 24 & 26 & 26 & 26 & 26 & 26.79 & 11 & 38.34 & 15 & 24.11 & 16 & 22.88 & 16 & 21.33 & 18 & 9.18 & 17 \\
RD3016 & 15 & 19 & 19 & 19 & 19 & 18 & 19 & 32.40 & 1 & 65.00 & 6 & 32.15 & 1 & 65.00 & 5 & 31.90 & 1 & 65.00 & 4 \\
NDB1173 & 14 & 11 & 12 & 12 & 11 & 12 & 12 & 27.35 & 10 & 65.51 & 5 & 27.10 & 8 & 65.54 & 4 & 26.88 & 7 & 66.90 & 3 \\
HUB273 & 27 & 29 & 29 & 29 & 28 & 28 & 28 & 26.75 & 12 & 32.79 & 16 & 26.21 & 10 & 30.90 & 15 & 25.63 & 9 & 30.29 & 14 \\
KB1815 & 35 & 32 & 33 & 33 & 34 & 35 & 34 & 25.69 & 17 & 25.63 & 17 & 23.60 & 17 & 13.84 & 18 & 21.41 & 17 & 3.97 & 18 \\
RD3015 & 27 & 18 & 19 & 19 & 19 & 19 & 19 & 25.80 & 16 & 53.37 & 9 & 25.30 & 14 & 51.74 & 8 & 24.80 & 13 & 51.85 & 8 \\
RD2552 & 21 & 22 & 22 & 22 & 21 & 19 & 20 & 26.11 & 15 & 50.54 & 12 & 25.88 & 11 & 50.63 & 9 & 25.62 & 10 & 52.09 & 7 \\
\hline
\end{tabular}


Maximum yield expressed by RD3016 followed by KB1822 and RD3017 as good variation had been observed from 32.4 to $22.2 \mathrm{q} / \mathrm{ha}$ among genotypes. Genotypic adaptability index expressed the higher values by RD3016 KB1822, RD3017 whereas Harmonic mean of genotypic values ranked RD3016, KB1822, RD3017 barley genotypes. Superiority index had observed lower value expressed by RD3017, RD2907, HUB274 and large value by KB1815.

\section{Biplot clustering pattern}

\section{First year of study 2018-19}

Loadings of studied measures as per first two significant principal components were reflected in table 6 . Biplot graphical analysis considered two PCAs accounted as $60.7 \%$ of variation of the stability measures accounted by both (Bocianowski et al., 2019). Studied measures grouped into three major clusters. MASV1 clubbed with ASTAB, EV, SIPC, Za, W3, WAASB and MASV measures (Fig 1). Yield based measures clubbed with corresponding SI measures. Measure IPCA1 and W2 maintained distance from measures and observed as outliers in different quadrant.

\section{Second year of study 2019-20}

Biplot graphical analysis based on first two significant principal component analysis (PCA) of the measures to explore the association if any among them (Fig 2). However,

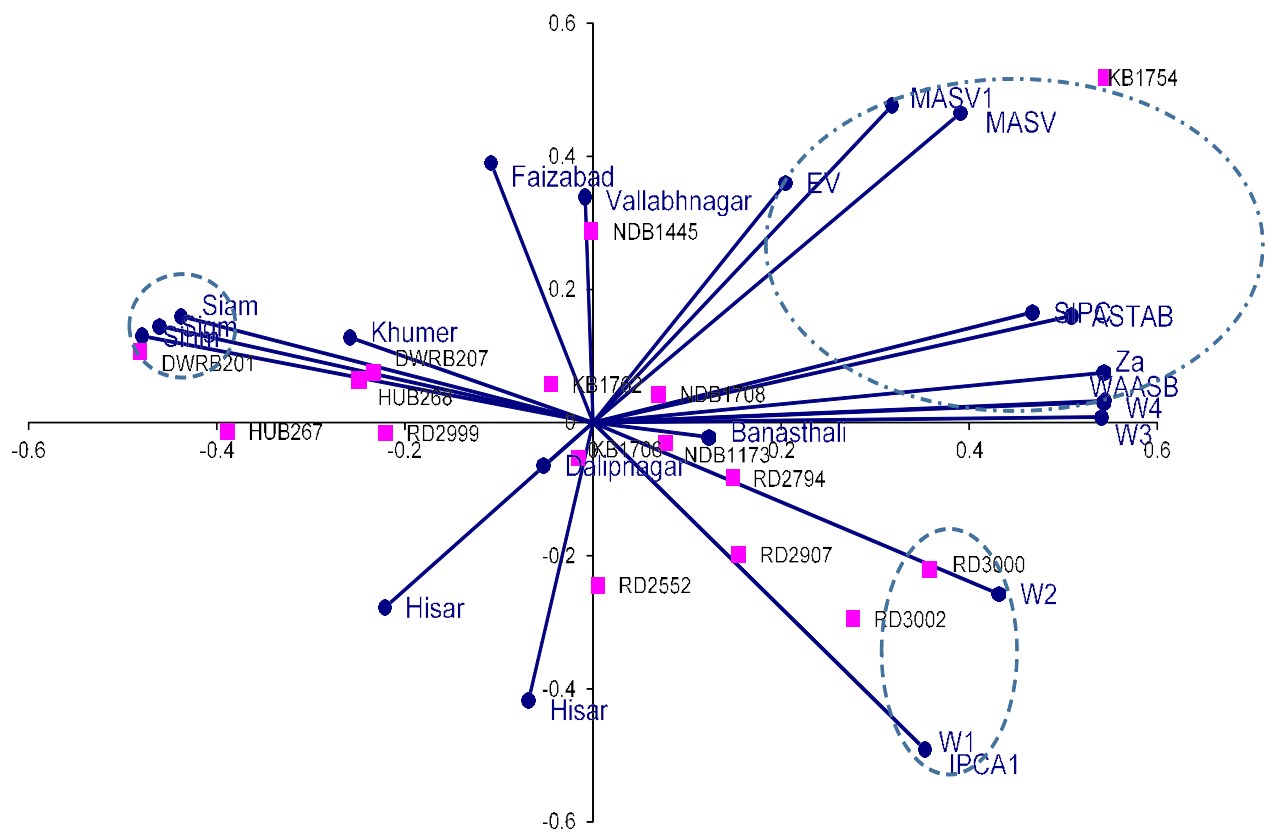

Fig 1: Biplot analysis of superiority index and other measures of barley genotypes 2018-19.

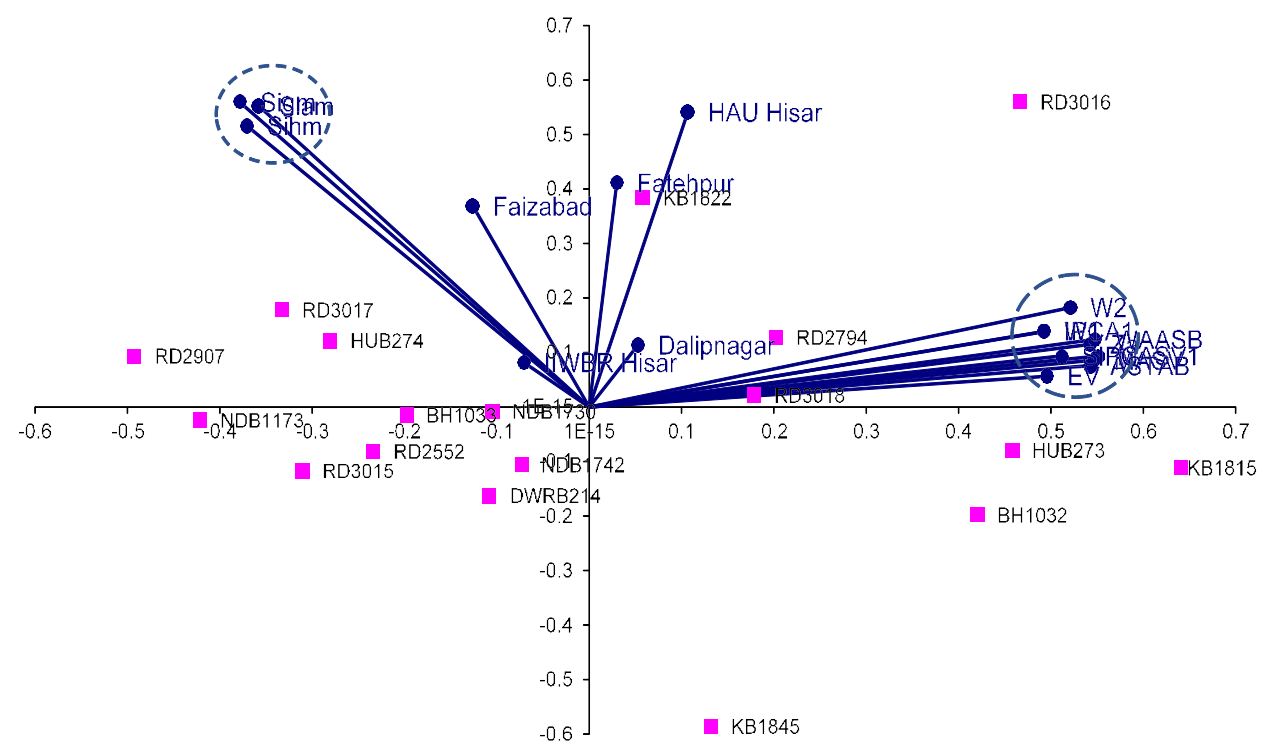

Fig 2: Biplot analysis of stability and adaptability measures of barley genotypes 2019-20. 
Table 10: Loadings of measures as per two principal components 2019-20.

\begin{tabular}{lcc}
\hline Measure & PC1 & PC2 \\
\hline IPCA1 & 0.2752 & 0.1081 \\
MASV1 & 0.3080 & 0.0731 \\
MASV & 0.3056 & 0.0671 \\
Za & 0.3031 & 0.0900 \\
EV & 0.2771 & 0.0441 \\
SIPC & 0.2861 & 0.0733 \\
ASTAB & 0.3034 & 0.0595 \\
W1 & 0.2752 & 0.1081 \\
W2 & 0.2913 & 0.1426 \\
WAASB & 0.3063 & 0.0970 \\
Siam & -0.2002 & 0.4322 \\
Sigm & -0.2115 & 0.4372 \\
Sihm & -0.2073 & 0.4034 \\
IIWBR Hisar & -0.0393 & 0.0636 \\
HAU Hisar & 0.0597 & 0.4226 \\
Dalipnagar & 0.0295 & 0.0889 \\
Faizabad & -0.0705 & 0.2888 \\
Fatehpur & 0.0167 & 0.3215 \\
71.76 & 56.87 & 14.89 \\
\hline
\end{tabular}

the loadings of the measures as per first two PCs were reflected in Table 10 . Nearly $71.8 \%$ of variation of the stability measures accounted by two PCAs. Two major groups of measures depicted in Fig 2. Large number of AMMI based measures clubbed together and separate cluster of SI was also observed. Superiority indexes depicted very strong association ship irrespective of average, geometric or harmonic values of genotypes. AMMI based measures and stability measures as per absolute values of scores tend to be of strong correlated pattern.

\section{CONCLUSION}

Simultaneous use of stability and yield would be more appropriate to recommend high-yielding stable genotypes. Advantages of AMMI and BLUP had been combined in Superiority Indexes to increase the reliability of multilocations trials analysis. The researchers may prioritize the productivity of a genotype rather than its stability (and viceversa) as per the goal of crop breeding trials.

\section{ACKNOWLEDGEMENT}

The barley genotypes were evaluated at research fields at coordinated centers of AICW \& BIP across the country. First author sincerely acknowledges the hard work of all the staff for field evaluation and data recording of genotypes.

\section{Conflict of interest}

No conflict of interest.

\section{REFERENCES}

Agahi, K., Ahmadi, J., Oghan, H.A., Fotokian, M.H., Orang, S.F. (2020). Analysis of genotype $\times$ environment interaction for seed yield in spring oilseed rape using the AMMI model. Crop Breeding and Applied Biotechnology. 20(1): e26502012.

Ajay, B.C., Bera, S.K., Singh, A.L., Kumar, N., Gangadhar, K., Kona, P. (2020). Evaluation of Genotype $\times$ Environment interaction and yield stability analysis in peanut under phosphorus stress condition using stability parameters of AMMI, odel. Agric. Res. 9: 477-486.

Bocianowski, J., Niemann, J., Nowosad, K. (2019). Genotype-by environment interaction for seed quality traits in interspecific cross-derived Brassica lines using additive main effects and multiplicative interaction model. Euphytica. 215(7): 1-13.

Farshadfar, E. (2008). Incorporation of AMMI stability value and grain yield in a single non-parametric index (GSI) in bread wheat. Pak. J. Biol. Sci. 11: 1791-1796.

Farshadfar, E., Mahmodi, N. and Yaghotipoor, A. (2011). AMMI stability value and simultaneous estimation of yield and yield stability in bread wheat (Triticum aestivum L.). Aust. J. Crop Sci. 5: 1837-1844.

Gauch, H.G. (2013). A simple protocol for AMMI analysis of yield trials. Crop Sci. 53: 1860-1869.

Olivoto, T., Lucio, A. Dal'Col, Gonzalez, Silva, J.A., da, Marchioro, V.S. (2019). Mean performance and stability in multienvironment trials I: Combining features of AMMI and BLUP techniques. Agron. J. 111: 1-12.

Oyekunle, M., Menkir, A., Mani, H., Olaoye, G., Usman, I.S., Ado, S. (2017). Stability analysis of maize cultivars adapted to tropical environments using AMMI analysis. Cereal Res. Commun. 45: 336-345.

Piepho, H.P., Mo"hring, J., Melchinger, A.E., Bu“chse, A. (2008). BLUP for phenotypic selection in plant breeding and variety testing. Euphytica. 161(1): 209-228.

Rao, A.R. and Prabhakaran, V.T. (2005). Use of AMMI in simultaneous selection of genotypes for yield and stability. Journal of the Indian Society of Agricultural Statistics. 59: 76-82.

Resende, M.D.V, Duarte, J.B. (2007). Precision and quality control in variety trials. Pesquisa Agropecuaria Tropical. 37: 182194.

Sneller, C.H., Kilgore-Norquest, L. and Dombek, D. (1997). Repeatability of yield stability statistics in soybean. Crop Science. 37 : 383-390.

Zali, H., Farshadfar, E., Sabaghpour, S.H., Karimizadeh, R. (2012). Evaluation of genotype $\times$ environment interaction in chickpea using measures of stability from AMMI model. Ann. Biol. Res. 3: 3126-3136.

Zobel, R. (1994). Stress Resistance and Root Systems. In Proceedings of the Workshop on Adaptation of Plants to Serious Stresses. 1-4 August. INTSORMIL Publication 94-2, Institute of Agriculture and Natural Recourses. Lincoln, USA: University of Nebraska. 